\title{
Bulk Membrane Retrieval in the Synaptic Terminal of Retinal Bipolar Cells
}

\author{
Matthew Holt, Anne Cooke, Minnie M. Wu, and Leon Lagnado \\ Medical Research Council Laboratory of Molecular Biology, Cambridge CB2 2QH, United Kingdom
}

The mechanism of bulk membrane uptake at the synapse remains poorly defined, although exocytosis of synaptic vesicles is followed by compensatory membrane retrieval into both small vesicles and large cisternas or vacuoles. We investigated bulk retrieval in the presynaptic terminal of retinal bipolar cells. Fluorescence imaging of the membrane dye FM1- 43 indicated that $\mathrm{Ca}^{2+}$-triggered exocytosis was followed by endocytosis into small vesicles and larger vacuoles that could be selectively labeled using large fluorescent dextrans. Disruption of actin filaments with cytochalasin D or latrunculin B inhibited the formation and transport of vacuoles, but exocytosis and endocytosis continued at normal rates. Bulk retrieval was linked to remodeling of the actin network, and both processes were inhibited by 2-(4-morpholinyl)-8-phenyl-4H-1-benzopyran-4-one, an inhibitor of phosphatidylinositol 3-kinase (PI 3-kinase). The regulation of F-actin dynamics by $\mathrm{Ca}^{2+}$ and PI 3-kinase therefore played an important role in compensatory endocytosis at this synapse, but this role was confined to bulk membrane uptake. Capacitance measurements demonstrated that fast endocytosis and refilling of the rapidly releasable pool of vesicles were not dependent on F-actin or PI 3-kinase activity. The basic properties of bulk membrane retrieval at this synapse were very similar to macropinocytosis described in non-neural cells. Bulk retrieval did not play an essential role in maintaining the vesicle cycle during maintained stimulation, but we suggest that it may play a role in the structural plasticity of this synaptic terminal.

Key words: synapse; endocytosis; macropinocytosis; actin; phosphatidylinositol 3-kinase; calcium

\section{Introduction}

The release of neurotransmitter at the synapse involves the $\mathrm{Ca}^{2+}$ triggered fusion of small vesicles with the surface membrane (Takei et al., 1996; Gad et al., 1998; Richards et al., 2000; Teng and Wilkinson, 2000). To maintain normal synaptic transmission, the excess membrane must be efficiently retrieved (Koenig and Ikeda, 1989). Three basic mechanisms of compensatory endocytosis have been proposed to operate at the presynaptic terminal. The first two, clathrin-mediated endocytosis and "kiss-and-run," involve retrieval of small vesicles. During clathrin-mediated endocytosis, clathrin-coated invaginations of the surface membrane are internalized and the clathrin coat is removed before the next round of exocytosis (for review, see Slepnev and De Camilli, 2000). In contrast, the kiss-and-run model proposes that vesicles do not fully collapse into the surface membrane, but recycle very rapidly after closure of a fusion pore that allows escape of the neurotransmitter. These schemes do not account for the fact that large cisternas formed directly from the plasma membrane have been observed consistently at synapses that have been stimulated strongly (Fried and Blaustein, 1978; Takei et al., 1996; Richards et al., 2000; Teng and Wilkinson, 2000) or after endocytosis has been transiently blocked (Koenig and Ikeda, 1989, 1996). A third model has therefore been proposed in which small vesicles are retrieved directly from the plasma membrane, in parallel with large cisternas formed by bulk membrane retrieval. Synaptic vesicles can also be formed from these larger compartments (Takei et al., 1996; Gad et al., 1998; Richards et al., 2000; Teng and Wilkin-

Received Sept. 9, 2002; revised Nov. 13, 2002; accepted Dec. 5, 2002.

We thank Artur Llobet and Vahri Beaumont for the discussions of this work.

Correspondence should be addressed to Leon Lagnado, Medical Research Council Laboratory of Molecular Biol-

ogy, Hills Road, Cambridge CB2 2QH, UK. E-mail: LL1@mrc-Imb.cam.ac.uk.

Copyright $\odot 2003$ Society for Neuroscience $\quad$ 0270-6474/03/231329-11\$15.00/0 son, 2000). A recent study of motor nerve terminals has demonstrated that these two recycling routes refill functionally distinct vesicle pools; vesicles directly retrieved from the plasma membrane quickly become available for release, whereas vesicles derived from bulk membrane retrieval are generated more slowly and refill a reserve pool (Richards et al., 2000). A large number of observations, therefore, indicate that membrane recycling at the synapse involves direct retrieval of small vesicles and bulk uptake of membrane.

The molecular mechanisms of bulk membrane retrieval at the synapse are still unknown. In non-neural cells, retrieval of large membrane compartments does not involve clathrin-coated intermediates but does involve the actin cytoskeleton and its modulation by phosphoinositides (Lamaze and Schmid, 1995; Araki et al., 1996; Nichols and Lippincott-Schwartz, 2001). A number of observations have suggested that phosphoinositides and actin might also play a role in endocytosis at the synapse (Qualmann et al., 2000; Slepnev and De Camilli, 2000; Cremona and De Camilli, 2001; Osborne et al., 2001). For instance, phosphoinositides are involved in the formation of clathrin coats (Ford et al., 2001) and the nucleation of an actin-based cytoskeleton at endocytic sites (Gad et al., 2000). The actin cytoskeleton may then interact with the endocytic machinery through molecules such as syndapin (Qualmann and Kelly, 2000). Additionally, a polyphosphoinositide phosphatase, synaptojanin, has been found to modulate endocytosis and the actin cytoskeleton at the reticulospinal synapse of lamprey (Gad et al., 2000).

To test directly whether actin or phosphoinositides are involved in any form of endocytosis at the synapse, we investigated compensatory membrane retrieval in the giant synaptic terminal of retinal bipolar cells using fluorescence microscopy and capacitance measurements. We found that there were at least two 
membrane compartments that could be distinguished morphologically and functionally: small vesicles and larger vacuolar structures. The formation and movement of the larger compartment required the activity of phosphatidylinositol 3-kinase (PI 3-kinase) and actin polymerization, whereas the retrieval of small vesicles did not. The properties of bulk membrane retrieval at this synapse were very similar to macropinocytosis, an actindependent mechanism of endocytosis observed in a number of non-neural cells and especially well characterized in macrophages and dendritic cells (Swanson and Watts, 1995; Araki et al., 1996; West et al., 2000). Capacitance measurements indicated that basic aspects of synaptic function were unaffected by actin, including fast exocytosis, fast endocytosis, and refilling of the rapidly releasable pool (RRP) of vesicles. These results demonstrate that activity-dependent regulation of actin dynamics plays a key role in compensatory endocytosis at the ribbon synapse of bipolar cells, but that this role is confined to bulk membrane retrieval by macropinocytosis. Although macropinocytosis was strictly dependent on the stimulation of exocytosis, it was not essential for maintaining the vesicle cycle. We suggest that macropinocytosis may play a role in the structural plasticity of this synaptic terminal.

\section{Materials and Methods}

Isolation and stimulation of depolarizing bipolar cells. Depolarizing bipolar cells were acutely dissociated from the retinas of goldfish using methods described previously (Lagnado et al., 1996). The normal Ringer's solution contained the following (in $\mathrm{mM}$ ): $120 \mathrm{NaCl}, 2.5 \mathrm{KCl}, 1 \mathrm{MgCl}_{2}$, $2.5 \mathrm{CaCl}_{2}, 10$ glucose, 10 HEPES, pH 7.3, $280 \mathrm{mOsm} / \mathrm{kg}^{-1}$. To stimulate continuous vesicle cycling, cells were depolarized in Ringer's solution containing $50 \mathrm{~mm} \mathrm{KCl}$, prepared by iso-osmotic replacement of $\mathrm{NaCl}$. The $0 \mathrm{Ca}^{2+}$ solutions were made by omitting $\mathrm{CaCl}_{2}$ and adding $1 \mathrm{~mm}$ EGTA. The osmolarity of the $0 \mathrm{Ca}^{2+}$ solution was $275 \mathrm{mOsm} / \mathrm{kg}^{-1}$. Bipolar cells were depolarized in this $0 \mathrm{Ca}^{2+}$ solution for $2 \mathrm{~min}$ in all experiments, before the application of $2.5 \mathrm{mM} \mathrm{Ca}^{2+}$.

All drugs were obtained from Calbiochem (La Jolla, CA) and maintained as stocks in DMSO. These stocks were diluted at least 1:1000 to achieve the final concentrations stated in Results. All drugs were applied for at least $15 \mathrm{~min}$ before an experiment.

Confocal microscopy. Confocal microscopy was performed using a BioRad (Hertfordshire, UK) Radiance 2000 scanhead mounted on a Nikon (Surrey, UK) TE300 inverted microscope with a $60 \times$ Nikon PlanApo oil objective (numerical aperture, 1.4). To monitor total membrane retrieval, FM1-43 (Molecular Probes, Eugene, OR) was applied at a concentration of $10 \mu \mathrm{M}$ while depolarizing the cell in $50 \mathrm{~mm} \mathrm{~K}+2.5 \mathrm{~mm}$ $\mathrm{Ca}^{2+}$. FM1-43 was imaged using the $488 \mathrm{~nm}$ line of the argon ion laser while collecting the emission through a $500 \mathrm{~nm}$ long-pass filter. The confocal iris was set to $2 \mathrm{~mm}$ and the laser power was typically $1.5 \%$. Simultaneous differential interference contrast (DIC) images were acquired to visualize membrane ruffling and the appearance of vacuoles. FM1-43 uptake was quantified as a percentage of the fluorescence measured when the surface membrane was stained under resting conditions (Lagnado et al., 1996). All results represent the mean \pm SEM.

Macropinosomes were selectively marked using a $40 \mathrm{kDa}$ dextran labeled with tetramethylrhodamine (Molecular Probes). The dextran was applied at a concentration of $50 \mu \mathrm{M}$, and the cell was depolarized in 50 $\mathrm{mm} \mathrm{K}{ }^{+} / 2.5 \mathrm{mM} \mathrm{Ca}^{2+}$ for $1 \mathrm{~min}$, after which time the dextran and $\mathrm{Ca}^{2+}$ were removed. Tetramethylrhodamine was excited using the $543 \mathrm{~nm}$ line of the helium-neon laser, and emitted light was collected through a 570 $\mathrm{nm}$ long-pass filter. To quickly measure fluorescence through most of the terminal, the confocal microscope was operated in a mode similar to conventional epifluorescence: the iris was opened to its maximum diameter, and the "multiphoton" lens was inserted in the scanhead to collect light from the thickest possible optical section. Uptake of the dextran was localized to the terminal and absolutely dependent on $\mathrm{Ca}^{2+}$ influx. Loading was quantified as the fluorescence intensity per unit area of the terminal (after background subtraction). Image processing was performed using IPLab (Signal Analytics, Fairfax, VA) or LaserPix (Bio-Rad) and then analyzed further using Igor Pro (Wavemetrics, Lake Oswego, OR). All results represent the mean \pm SEM.

Analysis of macropinosome numbers and movements. For counting, macropinosomes were loaded with $40 \mathrm{kDa}$ dextran, and a $z$-series of images was collected at intervals of $0.5 \mu \mathrm{m}$. The $z$-series was median filtered $(3 \times 3$ pixels $)$ and used to construct three-dimensional projections using IPLab software. All macropinosomes discernable from the top view were marked, tilting the projection to enable individual spots to be distinguished. The count was repeated for the opposite view, and the mean of the two counts was taken as the number per terminal. To measure their size, macropinosomes were identified by referring to the marks in the projection and relocated in the original $z$-series; their diameter was measured in the plane containing the largest section. When macropinosomes were not spherical, a mean was taken of major and minor axes.

To measure their movements, macropinosomes were loaded with 40 $\mathrm{kDa}$ dextran labeled with tetramethylrhodamine, and their distribution was assessed as the mean distance from the plasma membrane. A series of 16-32 lines originating from the geometric center of the terminal was superimposed on the fluorescence images, and the intensity profile of each line was measured. The same lines were also superimposed on the DIC image, and the location of the plasma membrane was marked by eye. Fluorescence intensity profiles were then aligned to the position of the plasma membrane and averaged to obtain the mean distribution of macropinosomes in a radial direction from the plasma membrane. The mean distance was defined as the position of the first peak in the fluorescence profile. Movements of macropinosomes were quantified by repeating this measurement on a series of images obtained at $1 \mathrm{~min}$ intervals.

Phalloidin staining of F-actin. Cells were plated onto coverslips and incubated in various test solutions for $6 \mathrm{~min}$ before fixing for $10 \mathrm{~min}$ in ice-cold Ringer's solution containing $0.25 \%$ glutaraldehyde (Sigma, Dorset, UK). Cells were then permeabilized with $0.5 \%$ Triton X-100 (BDH Chemicals, Dorset, UK) in "cytoskeletal buffer" for $2 \mathrm{~min}$ in the presence of $0.06 \%$ glutaraldehyde. Cytoskeletal buffer was used for all steps after permeabilization and contained (in $\mathrm{mm}$ ): $137 \mathrm{NaCl}, 5 \mathrm{KCl}, 1$ $\mathrm{Na}_{2} \mathrm{HPO}_{4}, 0.4 \mathrm{KH}_{2} \mathrm{PO}_{4}, 5.5$ glucose, $4 \mathrm{NaHCO}_{3}, 2 \mathrm{MgCl}_{2}, 2 \mathrm{EGTA}$, and 10 MES, pH 6.0. After removing the Triton X-100 solution, cells were then fixed with $0.25 \%$ glutaraldehyde for $10 \mathrm{~min}$. After washing for $5 \mathrm{~min}$ in cytoskeletal buffer, any remaining fixative was quenched with fresh sodium borohydride $(0.5 \mathrm{mg} / \mathrm{ml}$; Sigma) for $15 \mathrm{~min}$ on ice. Coverslips were then incubated in 10\% normal goat serum (Vector Laboratories, Burlingame, CA) for $15 \mathrm{~min}$ at room temperature before inversion onto $0.5 \mu \mathrm{M}$ Oregon Green 488 phalloidin (Molecular Probes) for $40 \mathrm{~min}$ at room temperature. Coverslips were then washed three times and mounted on ProLong antifade media (Molecular Probes). Confocal images of the actin network after various drug treatments were obtained using equivalent laser powers and gains with an iris aperture of $3 \mathrm{~mm}$.

The extension of the F-actin network under the plasma membrane, which occurred in response to maintained depolarization, was quantified using the method described by Job and Lagnado (1998). Briefly, the intensity profile was plotted along a line drawn across the center of the terminal, perpendicular to the plasma membrane. This profile showed two peaks corresponding to the high density of cortical F-actin under the plasma membrane at opposite sides of the terminal. The plasma membrane was taken as the point at which the intensity of phalloidin staining was two SDs greater than the background. The inner limit of the peak was defined as the point at which the intensity was two SDs greater than the mean intensity in the central region of the synaptic terminal. The distance between these two points was taken as the depth of the network. Thus, two measurements were obtained from each line profile on a single terminal.

Electrophysiology. Capacitance measurements were made from synaptic terminals that had detached from the axon during dissociation. Terminals were whole-cell voltage-clamped, and capacitance measurements were performed as described by Neves and Lagnado (1999). The solution in the patch pipette contained (in $\mathrm{mM}$ ): 110 cesium methane-sulfonate, 5 $\mathrm{MgCl}_{2}, 3 \mathrm{Na}_{2} \mathrm{ATP}, 1 \mathrm{Na}_{2}$ GTP, 10 TEA-Cl, 0.4 BAPTA, and 20 HEPES, pH $7.2,260 \mathrm{mOsm} / \mathrm{kg}^{-1}$. Electrode resistances were 3-6 M $\Omega$; the input resistance of the terminals was typically $1-10 \mathrm{G} \Omega$ at $-70 \mathrm{mV}$. 

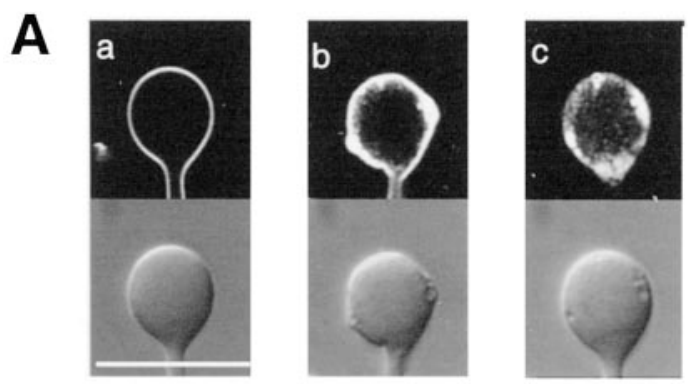

\section{B}
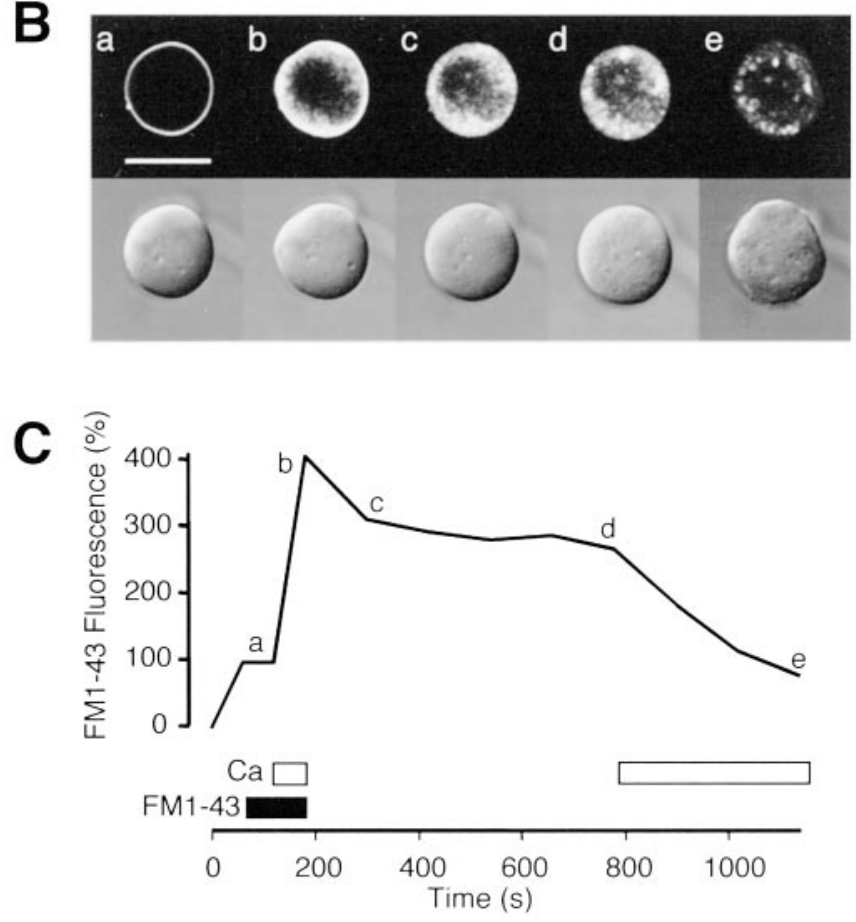

Figure 1. Continuous vesicle cycling was accompanied by membrane retrieval into two functionally distinct compartments. $A$, Simultaneously obtained pairs of DIC and fluorescence images of a single synaptic terminal. In the absence of $\mathrm{Ca}^{2+}, \mathrm{FM} 1-43$ stained only the plasma membrane of a cell depolarized in $50 \mathrm{~mm} \mathrm{~K}^{+}(a)$. Addition of $2.5 \mathrm{~mm} \mathrm{Ca}^{2+}$ stimulated exocytosis and endocytosis, causing an increase in fluorescence. FM1- 43 uptake was also associated with membrane ruffling and the appearance of vacuoles in DIC images $(b, c)$. Scale bar, $10 \mu \mathrm{m}$. $B$, Vacuoles did not release their contents during maintained stimulation. Vesicle cycling was stimulated for $1 \mathrm{~min}$ in the presence of FM1-43(b) and then stopped by removing $\mathrm{Ca}^{2+}$ and FM1-43 (c). The DIC images show the appearance of many small vacuoles (compare $a, e$ ). In the absence of $\mathrm{Ca}^{2+}$, the fluorescence was constant for several minutes $(d)$, but reapplication of $\mathrm{Ca}^{2+}$ caused immediate destaining because of exocytosis, leaving large compartments that retained the dye $(e)$. Scale bar, $10 \mu \mathrm{m}$. C, Total fluorescence uptake in the synaptic terminal shown in $B$. The letters $a-e$ mark the timing of the corresponding images.

\section{Results}

Membrane retrieval into two functionally

\section{distinct compartments}

Retinal photoreceptors and bipolar cells respond to light with graded and sustained changes in membrane potential that regulate the tonic release of neurotransmitter (Dowling, 1987). Using FM1-43, it has been shown that tonic release from retinal bipolar cells is supported by a continuous and balanced cycle of exocytosis and endocytosis that occurs in response to maintained depolarization (Lagnado et al., 1996; Rouze and Schwartz, 1998). To investigate the nature of the endocytic compartments taking up FM1-43, we used a combination of DIC microscopy and fluorescence confocal microscopy, as shown in Figure $1 A$. To apply a maintained depolarizing stimulus mimicking the effect of light, the cell was perfused with medium containing 50 mM K ${ }^{+}$(Lagnado et al., 1996; Rouze and Schwartz, 1998; Zenisek et al., 2000). In the absence of external $\mathrm{Ca}^{2+}, \mathrm{FM} 1-43$ stained the surface membrane but was not taken up into the cell (Fig. $1 \mathrm{Aa}$ ). Stimulation of vesicle cycling during a 1 min application of $2.5 \mathrm{mM} \mathrm{Ca}^{2+}$ caused ruffling of the surface, accumulation of vacuoles in the periphery of the terminal, and uptake of FM1-43 (Fig. 1 $A b$ ). After removal of FM1-43 and $\mathrm{Ca}^{2+}$, vacuoles visible under DIC coincided with areas of particularly dense FM1-43 fluorescence, indicating that they contained membrane recently retrieved from the surface (Fig. 1Ac). FM1-43 was also taken up in a weaker diffuse pattern that did not obviously coincide with vacuoles (Fig. $1 A c$ ).

The diffuse fluorescence appeared to represent small vesicles loaded with dye, because it rapidly declined when vesicle cycling was stimulated after removing FM1-43 from the bathing medium. An example of this behavior is shown in Figure 1, $B$ and $C$. First, vesicle cycling was stimulated for $1 \mathrm{~min}$ in the presence of FM1-43 (Fig. $1 \mathrm{Bb}$ ). After $\mathrm{Ca}^{2+}$ and FM1-43 were removed, the fluorescence was constant for several minutes (Fig. $1 B c, d$ ), but reapplication of $\mathrm{Ca}^{2+}$ caused immediate destaining attributable to exocytosis (Fig. $1 \mathrm{Be}, \mathrm{C}$ ). The loss of FM1-43 was not uniform; the dye was primarily lost from the diffuse compartment, causing the bright puncta to become more prominent (Fig. $1 \mathrm{Be}$ ). Similar behavior was observed in $>40$ cells.

\section{Large compartments were selectively labeled with large dextrans}

FM1-43 labels all endocytic compartments because it is soluble in membrane. To selectively label larger compartments, we used large fluid-phase markers that have restricted access to small vesicles (Berthiaume et al., 1995; Araki et al., 1996). The loading of vacuoles with a $40 \mathrm{kDa}$ dextran labeled with tetramethylrhodamine is shown in Figure 2A. The cell was continuously depolarized, and membrane cycling was stimulated for $1 \mathrm{~min}$ by applying 2.5 $\mathrm{mm} \mathrm{Ca}{ }^{2+}$ in a solution containing $50 \mu \mathrm{M}$ dextran (Fig. 2Ab). Uptake of the dextran was confined to the synaptic terminal (data not shown), and the staining was dominated by "hot-spots," many of which coincided with vacuoles visible under DIC (Fig. $2 A c)$. Two observations indicated that small vesicles were not accessible to this large dextran: (1) the diffuse membrane compartment labeled by FM1-43 was not labeled by the dextran, and (2) the dextran that was taken up could not be released when exocytosis was stimulated for several minutes (Fig. 3). Together, the results in Figures 1-3 indicate that continuous exocytosis was followed by membrane retrieval into two distinct compartments: small vesicles that became available for a second round of release and large vacuolar compartments that did not take part in the continuous vesicle cycle.

The frequency and size of vacuoles loaded with dextrans were estimated by taking a series of confocal sections at different depths through the terminal (Fig. 2B) (see Materials and Methods). The number of vacuolar structures per terminal formed after 1 min of stimulation averaged $29 \pm 13$ (mean \pm SD; four terminals). This number is likely to represent a minimum, because large compartments were easier to detect than small ones. The size of these compartments varied widely, with apparent diameters ranging from a fraction of a micron to $1-2 \mu \mathrm{m}$ (median of $0.7 \mu \mathrm{m}$ ) (Fig. $2 C$ ). It is possible that smaller compartments only appeared spherical because of the limited spatial resolution of the confocal microscope. The large, heterogeneous size of these compartments was similar to macropinosomes formed at areas of 
A

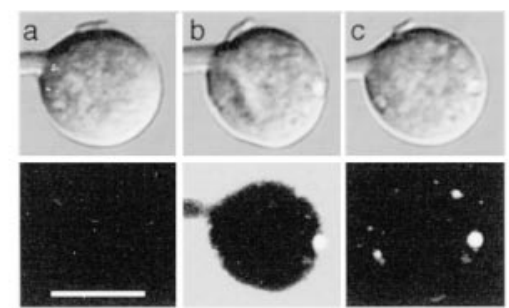

B
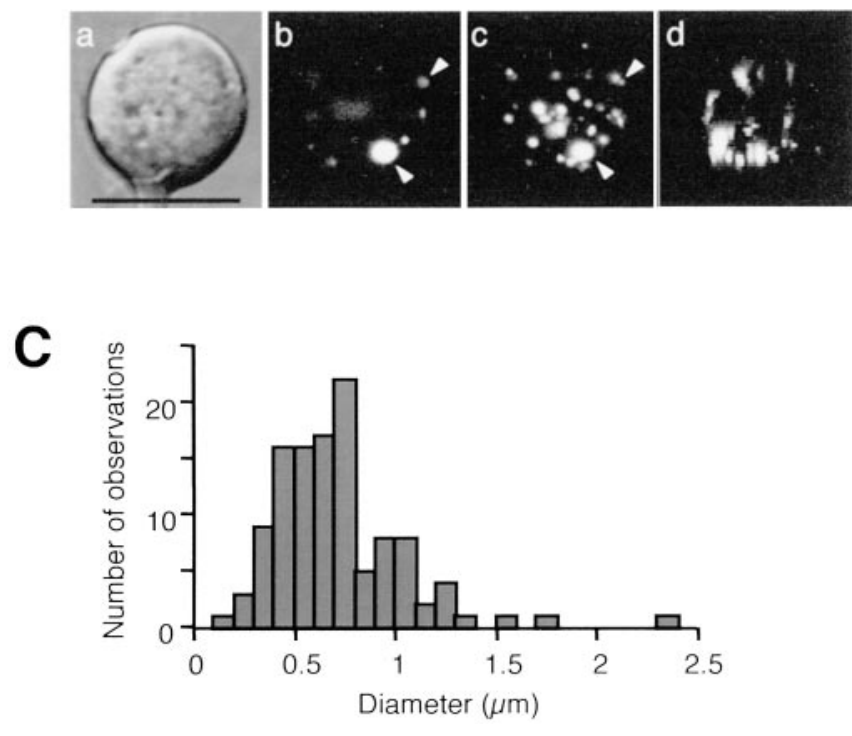

Figure 2. Vacuoles were preferentially labeled by large fluorescent dextrans. $A$, Pairs of DIC and fluorescence images. The cell was continuously depolarized in $50 \mathrm{~mm} \mathrm{~K}^{+}(a)$ and then 50 $\mu \mathrm{m}$ tetramethylrhodamine-labeled $40 \mathrm{kDa}$ dextran and $2.5 \mathrm{~mm} \mathrm{Ca}^{2+}$ applied for $1 \mathrm{~min}$. The images in $b$ were acquired during washout of the dextran from the external medium; vacuoles apparent in the DIC image colocalize with bright fluorescent regions. Image c shows internalized marker after complete washout of the dextran. Scale bar, $10 \mu \mathrm{m}$. B, Counting vacuoles loaded with tetramethylrhodamine-labeled $40 \mathrm{kDa}$ dextran. $b$, A single confocal section through the middle of the terminal; $c$ is a projection from a series of z-sections through the entire terminal. $d$, A projection of the same terminal looking from the side, with the coverslip toward the bottom of the figure. The diameter of a vacuole was measured in the plane where it appeared largest; the arrowheads in $b$ show two vacuoles measured in this confocal section (diameters are 2.3 and $0.8 \mu \mathrm{m}$ ). The same two vacuoles are marked in the projection of the $z$-series shown in c. A total of 39 vacuoles were counted in this projection. Scale bar, $10 \mu \mathrm{m}$. C, Histogram showing the size distribution of dextran-labeled vacuoles formed in the first minute of stimulation. Collected results from four cells that together contained a total of 115 vacuoles are shown.

membrane ruffling in macrophages and fibroblasts (Swanson and Watts, 1995). We should emphasize that these observations do not rule out the possibility that bulk retrieval also involves compartments $<0.5 \mu \mathrm{m}$ that were not reliably detected by our measurements of dextran uptake.

Large compartments formed directly from the surface membrane but did not remain attached

Three lines of evidence indicated that vacuoles observed in the terminal of bipolar cells were formed by bulk retrieval of surface membrane rather than fusion of small vesicles within the terminal. First, DIC images often showed large vacuoles forming directly at the surface (Fig. 1A). Second, vacuoles became loaded with large dextrans from the external medium that are generally thought to be too large to enter small vesicles (Figs. 2, 3). Third,
FM1-43 taken up into small vesicles did not appear in vacuoles (Fig. 4). This last observation was made when retrieved membrane was stained with FM1-43 after the formation of vacuoles. Terminals were first stimulated for $3 \mathrm{~min}$ in the absence of FM143 , and then FM1-43 was added to the external medium to stain membrane retrieved subsequently. The example shown in Figure $4 \mathrm{~A}$ demonstrates that the diffuse fluorescence associated with small vesicles was undetectable in the vacuoles visible under DIC, indicating that vesicles did not exchange their contents with larger compartments, at least on the timescale of minutes.

Cisternal compartments at the neuromuscular junction often appear attached to the surface membrane by thin tubules (Teng et al., 1999; Richards et al., 2000). Three observations strongly suggest that large compartments formed in the terminal of bipolar cells were completely disconnected from the surface. First, large compartments did not take up FM1-43 if the dye was added after they had been formed, indicating that they were no longer attached to the surface (Fig. 4). Second, FM1-43 washed out from the surface membrane within seconds of its removal from the external medium, yet remained in internal compartments for 10-15 min (see Figs. 1, 9). We expect that thin tubular structures connecting large compartments to the surface would have allowed FM1-43 to wash out within seconds, because FM1-43 diffuses in the membrane of bipolar cells very rapidly (Zenisek et

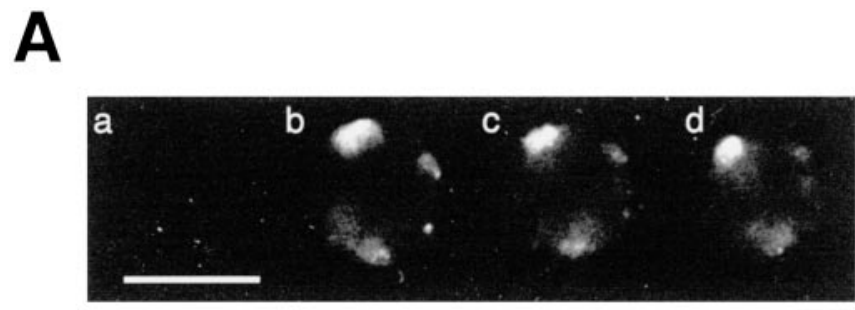

B

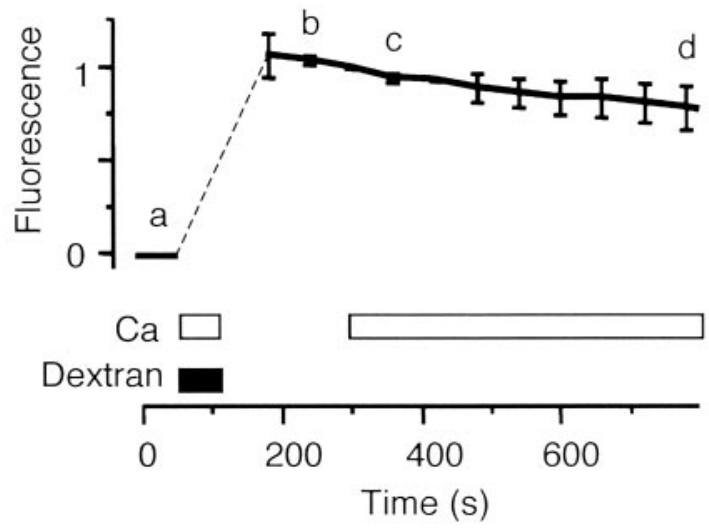

Figure 3. Dextrans were not released from vacuoles during maintained stimulation. $A$, Fluorescence images were obtained using a confocal microscope. Before dextran application, no fluorescence was associated with the synaptic terminal ( $a$ ). Membrane cycling was stimulated for 1 min in the presence of a $40 \mathrm{kDa}$ dextran and then stopped by removing $\mathrm{Ca}^{2+}$ and dextran (b). Reapplication of $\mathrm{Ca}^{2+}$ did not cause any appreciable loss of fluorescence $(c, d)$. In this example, total dextran uptake has been quantified by using the microscope in a nonconfocal mode to maximize light collection (see Materials and Methods). B, Fluorescent dextran uptake in bipolar cells is shown. The letters mark the timing of the corresponding images in $A$. Between 60 and $180 \mathrm{sec}$ the fluorescence was saturating as a result of the dextran in the extracellular solution (thin dashed line). Data are averaged from three terminals. Scale bar, $10 \mu \mathrm{m}$. 
al., 2002). Third, the largest compartments visible under DIC appeared completely circumscribed and distinct from the surface membrane (Figs. 1, 2, 4).

\section{Large compartments formed during the initial stages of stimulation}

The design of the experiment shown in Figure 4 took advantage of the observation that vacuoles tended to form during the first minute or so of a maintained stimulus. Figure $5, A$ and $B$, shows that the labeling of large compartments by a $40 \mathrm{kDa}$ dextran was reduced in the fourth minute of continuous stimulation, when dextran uptake averaged $29 \pm 5 \%$ of that in the first minute. The reduction in dextran uptake at later times could not be explained by a decrease in the rate of vesicle cycling, because the total amount of membrane stained by FM1-43 was very similar in the first and fourth minutes of stimulation (Fig. $5 B$ ). This result is in agreement with previous demonstrations that the total rate of membrane cycling during maintained depolarization is constant over periods of many minutes (Lagnado et al., 1996). Retrieval into large compartments, therefore, occurred predominantly during the initial stages of maintained activity, whereas at later times retrieval into small vesicles accounted for a greater fraction of the membrane retrieved. We suggest that bulk membrane uptake occurring during the initial stages of stimulation might con-
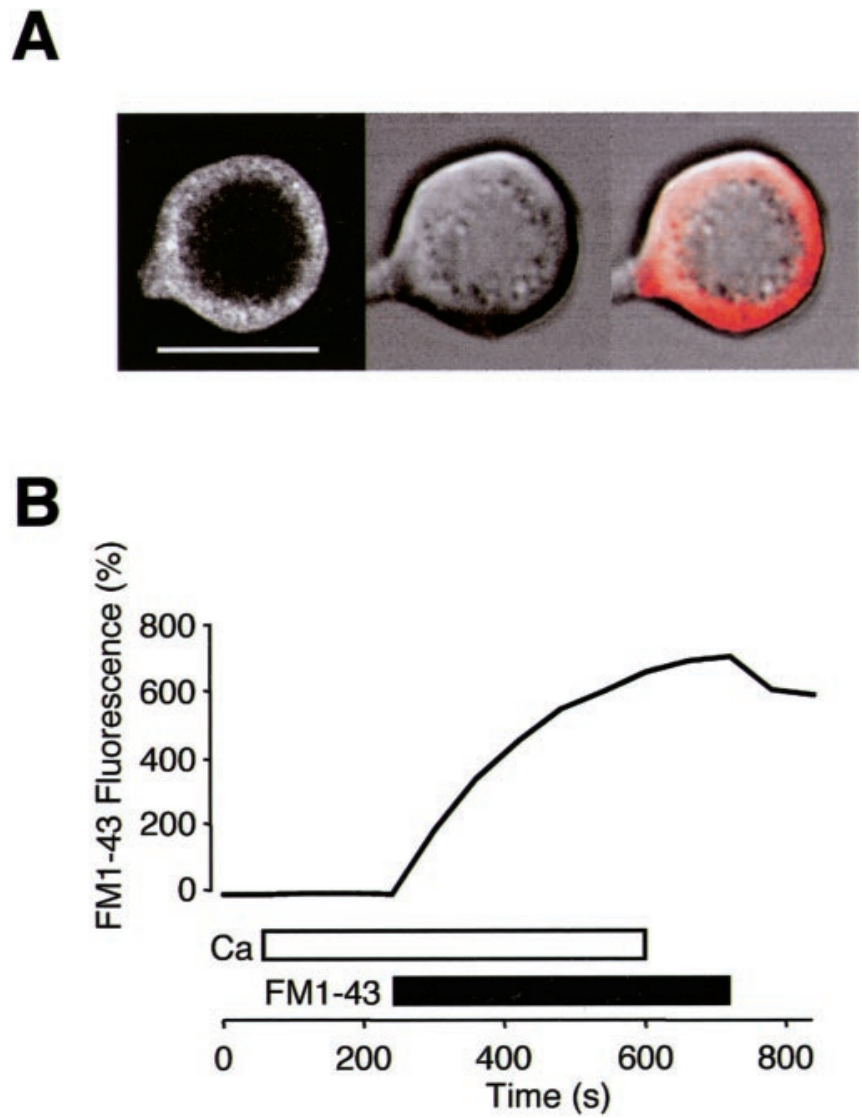

Figure 4. Vacuoles excluded the contents of retrieved vesicles. A, An example of an experiment in which a bipolar cell was continuously depolarized in the presence of $\mathrm{Ca}^{2+}$ and FM1- 43 applied after a delay of $3 \mathrm{~min}$. The images show the distribution of FM1-43 taken into the terminal (left), vacuoles under DIC (center), and an overlay of the two with fluorescence in red (right). Vacuoles are collected in a ring $\sim 2 \mu \mathrm{m}$ from the plasma membrane. Newly retrieved vesicles were localized to the region between the plasma membrane and vacuoles, but the vacuoles excluded FM1-43. Scale bar, $10 \mu \mathrm{m}$. B, Total fluorescence uptake in the synaptic terminal shown in $A$. Similar behavior was observed in five cells.
A 1st $\min$
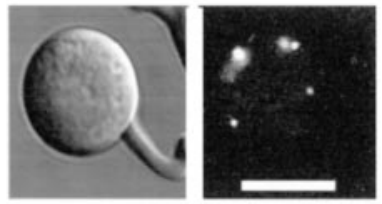

B

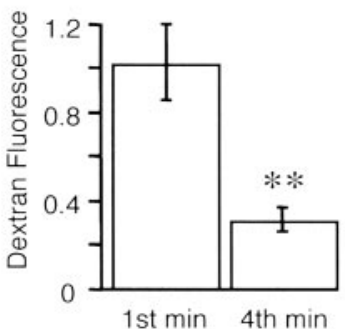

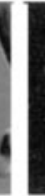

4th $\min$
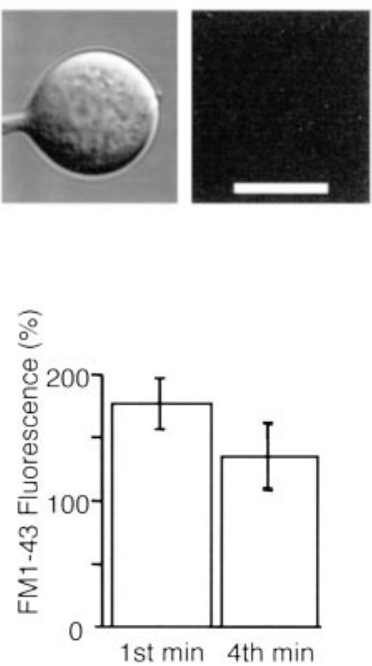

Figure 5. Bulk membrane retrieval occurred during the initial stages of maintained stimulation. $A$, Examples of dextran uptake after a $1 \mathrm{~min}$ application of the marker at the start of stimulation from rest (left) or 3 min after the beginning of stimulation (right). To maximize light collection, the confocal microscope was operated in a mode similar to normal epifluorescence (see Materials and Methods). Scale bars, $10 \mu \mathrm{m}$. B, Bulk membrane retrieval was significantly reduced in the fourth minute of stimulation compared with the first minute. The graph on the left shows total uptake of $40 \mathrm{kDa}$ dextran expressed relative to uptake in the first minute (1st $\min , n=17 ; 4$ th $\min , n=18$ ). In contrast, the graph on the right shows that the total FM1-43 uptake was similar during the first and fourth minutes of stimulation (1st min, $n=12$; 4th min, $n=5)$. $t$ test; ${ }^{* *} p<0.01$.

tribute to the structural plasticity of this synaptic terminal in the retina (see Discussion).

Bulk membrane retrieval was dependent on actin polymerization

The actin network in the synaptic terminal of bipolar cells is dynamic and regulated by $\mathrm{Ca}^{2+}$ (Job and Lagnado, 1998). In resting cells, F-actin was localized to small puncta at the plasma membrane (Fig. $6 A a, B$ ), but maintained stimulation caused the growth of a cortical F-actin network that extended to an average depth of $2.1 \mu \mathrm{m}$ from the plasma membrane (Fig. 6Ab,B) (Job and Lagnado, 1998). We tested whether growth of the actin network might be involved in bulk membrane retrieval, because such forms of endocytosis often involve actin polymerization (Lamaze and Schmid, 1995; Araki et al., 1996; Nichols and Lippincott-Schwartz, 2001). The extension of the actin network under the plasma membrane was quantified using the approach described by Job and Lagnado (1998) and described briefly in Materials and Methods. The stimulus-dependent growth of the cortical F-actin network was inhibited by $20 \mu \mathrm{M}$ latrunculin B (Fig. 6Ac,B) and $20 \mu \mathrm{M}$ latrunculin A (data not shown). These agents do not directly bind filamentous actin but act by sequestering actin monomers and preventing their polymerization (Morton et al., 2000). The disruption of actin filaments in the bipolar cell terminal by latrunculin B is therefore evidence that the drug blocked the normal function of monomeric actin. The F-actin network was also completely disrupted by application of $20 \mu \mathrm{M}$ cytochalasin $\mathrm{D}$, which prevents addition of actin monomers to filaments (data not shown) (Job and Lagnado, 1998). Latrunculin B and cytochalasin D also significantly reduced the uptake of a $40 \mathrm{kDa}$ dextran, indicating that actin polymerization was required for bulk membrane retrieval (Fig. 7A). The reduc- 
A

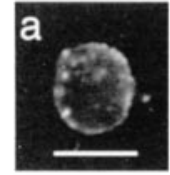

Unstim

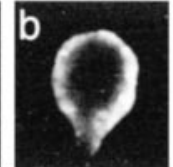

Control Stim

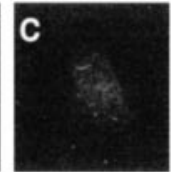

LatB

Stim

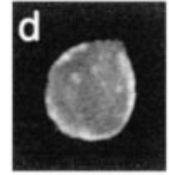
Stim
LY294002

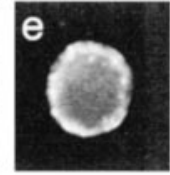

LY303511 Stim
B

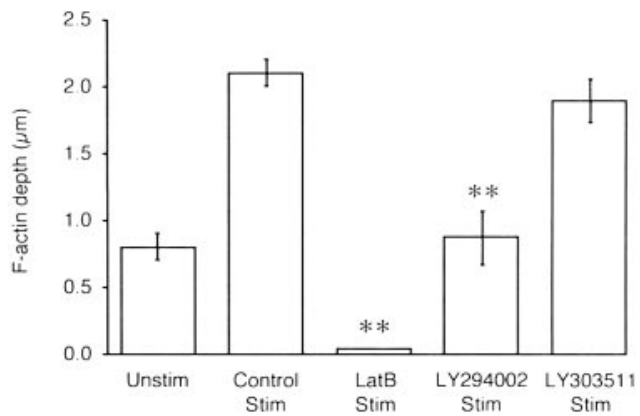

Figure 6. LY294002, a selective inhibitor of PI 3-kinase, suppressed the stimulus-dependent growth of cortical F-actin. A, F-actin stained with Oregon Green phalloidin in an unstimulated terminal $(a)$ and in terminals stimulated for 6 min under normal conditions $(b)$ and in the presence of $20 \mu \mathrm{m} \operatorname{LatB}(c), 50 \mu \mathrm{m} \operatorname{LY} 294002$ (d), or $50 \mu \mathrm{m} \operatorname{LY} 303511$ (e). Scale bar, $10 \mu \mathrm{m}$. $B$, Average depth of the cortical F-actin network under the conditions shown in A. Unstim, Unstimulated $(n=8)$; Stim, stimulated ( $n=8)$; latrunculin $B, n=8 ;$ LY294002, $n=12$; LY303511, $n=14$. $t$ test; ${ }^{* *} p<0.01$.

tion in dextran uptake after disruption of the actin network could not be explained by a reduced amount of exocytosis, because latrunculin B and cytochalasin D did not affect the rate of vesicle cycling measured using FM1-43 (Fig. 7B). An important deduction from this result is that when bulk membrane retrieval at the synapse is inhibited, other forms of endocytosis compensate effectively enough to maintain vesicle cycling at normal rates.

Bulk membrane retrieval was dependent on the activity of PI 3-kinase

In macrophages and cell lines, bulk membrane retrieval is dependent on the activity of PI 3-kinase, an enzyme that has also been implicated in vesicular membrane traffic (Clague et al., 1995; Araki et al., 1996). PI 3-kinase in neurons can be activated by $\mathrm{Ca}^{2+}$ influx during depolarization (Miller et al., 1997; Crowder and Freeman, 1999; Ikegami and Koike, 2000), so we tested its actions at the synapse of bipolar cells using 2-(4-morpholinyl)8-phenyl-4H-1-benzopyran-4-one (LY294002), a selective inhibitor of this enzyme (Vlahos et al., 1994). A $50 \mu \mathrm{M}$ concentration of LY294002 reduced the amount of dextran uptake by $90 \%$ (Fig. 7A), and $25 \mu \mathrm{M}$ LY294002 inhibited uptake by 50\% (data not shown). A $50 \mu \mathrm{M}$ concentration of 2-piperazinyl-8-phenyl4H-1-benzopyran-4-one (LY303511), a similar compound with little effect on PI 3-kinase (Vlahos et al., 1994), did not reduce dextran uptake (Fig. 7A). The total amount of membrane retrieved during maintained stimulation, as measured by FM1-43 uptake, was not significantly affected by treatment with $50 \mu \mathrm{M}$ LY294002 (Fig. 7B). Therefore, endocytosis into small compartments continued at normal rates when bulk membrane retrieval was blocked by LY294002, indicating that retrieval of vesicles was not dependent on the activity of PI 3-kinase. $\mathrm{Ca}^{2+}$-dependent
A

\section{Dextran}

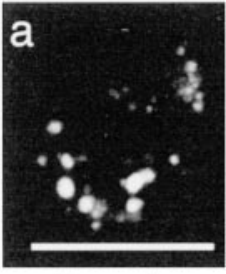

Control

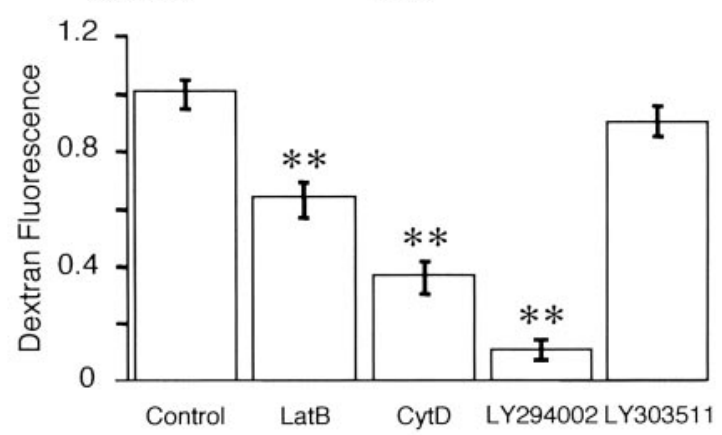

\section{B FM1-43}
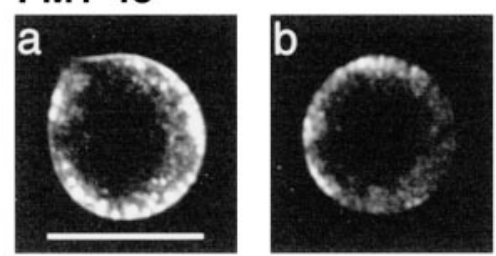

LatB

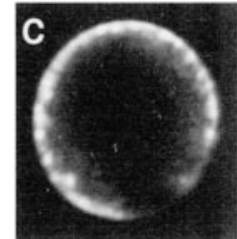

LY294002

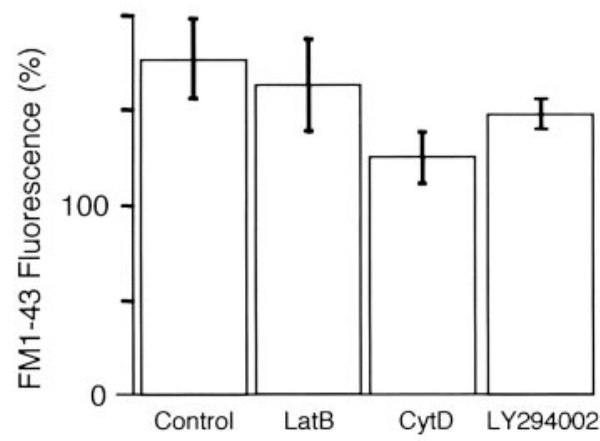

Figure 7. Agents disrupting cortical F-actin inhibited bulk membrane retrieval. A, Total uptake of tetramethylrhodamine $40 \mathrm{kDa}$ dextran after 1 min of stimulation in the absence $(a)$ or presence $(b)$ of $20 \mu \mathrm{m}$ LatB. These examples are projections of confocal $z$-series. Collected results are shown in the bar chart (bottom). The following significantly reduced bulk membrane retrieval (in $\mu \mathrm{M}$ ): 20 latrunculin B, 20 cytochalasin D, 50 LY294002. Control, $n=84$; latrunculin $\mathrm{B}, n=32$; cytochalasin D, $n=17 ; \mathrm{LY} 294002, n=13 ; \mathrm{LY} 303511, n=5 . t$ test; ${ }^{* *} p<0.01$. Scale bar, $10 \mu \mathrm{m}$. B, Uptake of FM1- 43 after 1 min of stimulation under normal conditions $(a)$ and in the presence of $20 \mu \mathrm{m}$ latrunculin $B(b)$ or $50 \mu \mathrm{m} \mathrm{LY} 294002$ ( $c$ ). The example images are single confocal sections taken through the middle of the terminal. Total FM1- 43 fluorescence was measured by epifluorescence (see Materials and Methods). Latrunculin B, cytochalasin D, and LY294002 did not significantly affect total membrane retrieval. Control, $n=12$; latrunculin B, $n=10$; cytochalasin D, $n=8$; LY294002, $n=5$. Scale bar, $10 \mu \mathrm{m}$.

growth of the cortical F-actin network was also inhibited by 50 $\mu \mathrm{M}$ LY294002 but not by $50 \mu \mathrm{M}$ LY303511 (Fig. 6).

The results in Figures 6 and 7 demonstrate that three drugs that inhibit or alter actin dynamics by different mechanisms 
(LY294002, latrunculin B, and cytochalasin D) all had the common effect of inhibiting bulk membrane retrieval without significantly affecting the total rate of membrane cycling. We conclude that bulk membrane retrieval in the synaptic terminal of bipolar cells is dependent on normal actin dynamics, but the retrieval of small vesicles is not.

The actin cytoskeleton was not involved in fast endocytosis or refilling of the rapidly releasable pool of vesicles

The experiments described above were performed on the timescale of tens of seconds, when exocytosis occurred continuously at a slow rate. Might membrane retrieval after a fast and transient burst of exocytosis occur by a different mechanism that is dependent on actin? This possibility is suggested by the large number of observations indicating that actin is involved in clathrinmediated endocytosis, both at the synapse (Qualmann et al., 2000; Slepnev and De Camilli, 2000; Cremona and De Camilli, 2001; Osborne et al., 2001) and in non-neural cells (Merrifield et al., 2002). We therefore tested the effects of actin-disrupting agents on fast modes of endocytosis measured in real time using the capacitance technique.

In the synaptic terminal of bipolar cells, there is an RRP of vesicles that can be completely released by a $20 \mathrm{msec}$ depolarization (Mennerick and Matthews, 1996; Burrone and Lagnado, 2000). The retrieval of these vesicles occurs by two kinetically distinct mechanisms; fast endocytosis has a time constant of $\sim 1-2$ sec, whereas slow endocytosis has a time constant of greater than $\sim 10 \mathrm{sec}$ (von Gersdorff and Matthews, 1994; Neves and Lagnado, 1999; Neves et al., 2001). Figure $8 A$ shows averaged responses to two $20 \mathrm{msec}$ stimuli delivered $10 \mathrm{sec}$ apart. Compared is the response in control conditions (DMSO alone) and in the presence of $20 \mu \mathrm{M}$ latrunculin B, $20 \mu \mathrm{M}$ cytochalasin D, or 50 $\mu \mathrm{M}$ LY294002. Figure $8 B$ shows these traces normalized to make the first response of fixed amplitude, allowing the kinetics of endocytosis to be directly compared. Looking at the responses to both stimuli, it can be seen that latrunculin B, cytochalasin D, and LY294002 did not significantly alter the kinetics of membrane retrieval. These results further support the conclusion that the actin cytoskeleton did not play an essential role in retrieval of vesicles at this synapse.

It has also been proposed that the actin cytoskeleton might regulate exocytosis at the synapse either by acting as a tether for the vesicles that are held in reserve (Hirokawa et al., 1989) or by preventing the maturation of docked vesicles into a fully primed state (Morales et al., 2000). Although latrunculin B and cytochalasin D completely disrupt the puncta of F-actin at the plasma membrane (Fig. 6Ac) (Job and Lagnado, 1998), neither had a significant effect on the amount of exocytosis triggered by the first $20 \mathrm{msec}$ stimulus (Fig. 8A), indicating that the actin cytoskeleton did not regulate the size of the RRP. The second 20 msec stimulus of the pair was applied to completely deplete the RRP a second time, allowing us to measure the degree to which it had refilled during the $10 \mathrm{sec}$ delay (Gomis et al., 1999). Under control conditions, the amount of exocytosis triggered by the second "emptying stimulus" was $\sim 50 \%$, indicating that half the rapidly releasable pool of vesicles had refilled in the $10 \mathrm{sec}$ interval. Figure $8, B$ and $C$, shows that latrunculin $\mathrm{B}$, cytochalasin $\mathrm{D}$, and LY294002 had no significant effect on refilling the RRP. We conclude that actin dynamics did not regulate the processes controlling the supply of new vesicles for rapid exocytosis at the active zone.

\section{A}

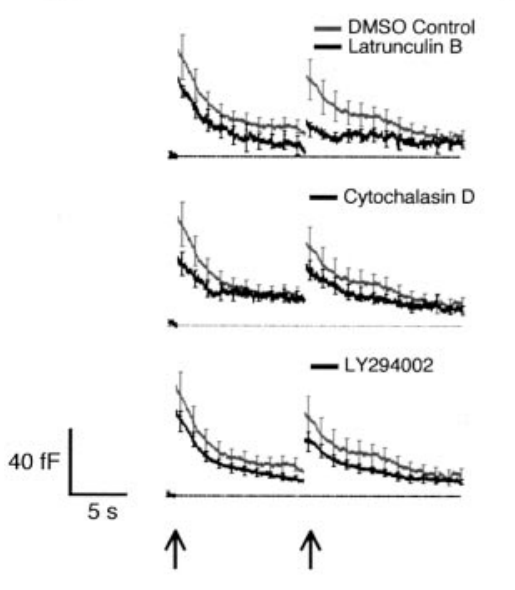

B

\section{C}

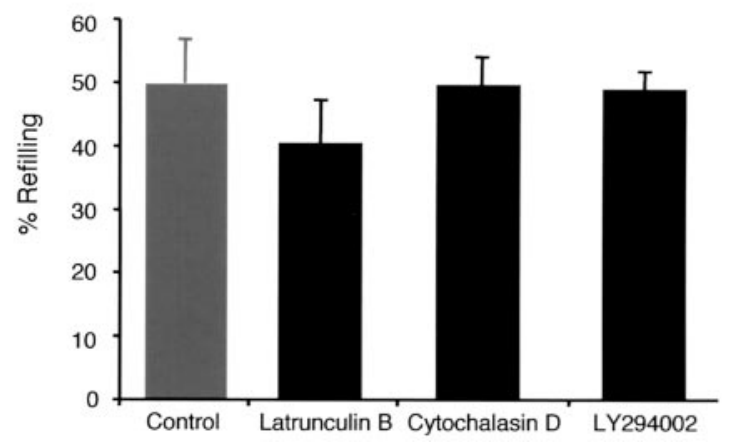

Figure 8. Actin depolymerizing agents and the PI 3-kinase inhibitor LY294002 had no effect on exocytosis, endocytosis, or the resupply of vesicles for exocytosis. $A$, Capacitance changes in response to two 20 msec depolarizations to $-10 \mathrm{mV}$ delivered 10 sec apart (arrows). Compared are averaged responses in DMSO alone (control) (in $\mu \mathrm{M}$ ): 20 latrunculin B, 20 cytochalasin D, and $50 \mathrm{LY} 294002$. The control responses are compared with each of the test conditions separately. Control, $n=5$; latrunculin B, $n=4$; cytochalasin D, $n=3 ; \mathrm{LY} 294002, n=5$. Error bars indicate 1 SEM. $B$, Traces in $A$ normalized to the peak of the first capacitance response to allow comparison of the timecourse of endocytosis. The falling phases of the traces superimpose closely. $C$, The response to the second stimulus expressed as a percentage of the first response provides a measure of the rate at which new vesicles were supplied for fast exocytosis. Latrunculin B, cytochalasin D, and LY294002 had no significant effect on vesicle resupply.

\section{$\mathrm{Ca}^{2+}$-dependent transport of large compartments driven by the actin cytoskeleton}

During maintained $\mathrm{Ca}^{2+}$ influx, the F-actin network extended radially from the plasma membrane up to a depth of $\sim 2 \mu \mathrm{m}$, and this occurred over a period of several minutes (Fig. 6) (Job and Lagnado, 1998). We found that vacuoles also showed a parallel movement away from the plasma membrane toward the center of the synaptic terminal. An example of this behavior is shown in Figure 9. Retrieved membrane compartments were stained by stimulating exocytosis for $1 \mathrm{~min}$ in the presence of FM1-43 (Fig. $9 A a-c)$. A second period of maintained stimulation caused release of FM1-43 from the diffuse compartment, but larger stained structures moved up to $2 \mu \mathrm{m}$ away from the plasma membrane toward the center of the terminal, so that twodimensional images showed a circle concentric with the plasma membrane (Fig. 9Ad,e). This behavior could also be observed under DIC (Fig. $4 A$ ).

The movement of these large compartments was measured by selectively labeling them with $40 \mathrm{kDa}$ dextran. The example in Figure $10 \mathrm{~A}$ shows vacuoles formed immediately after $1 \mathrm{~min}$ of stimulation $(a)$, then after $2 \mathrm{~min}$ in $0 \mathrm{Ca}(b)$, and finally after an 
A

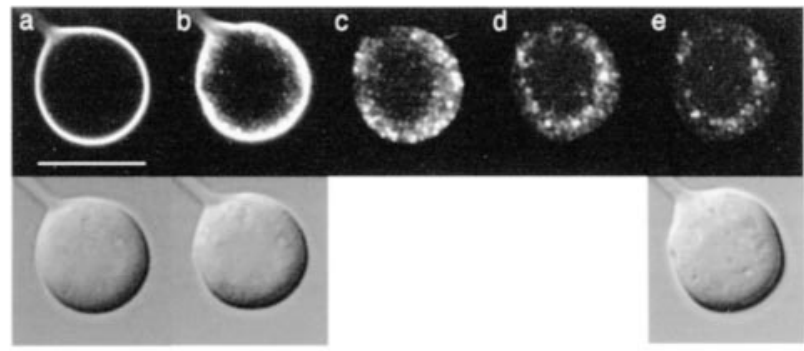

B

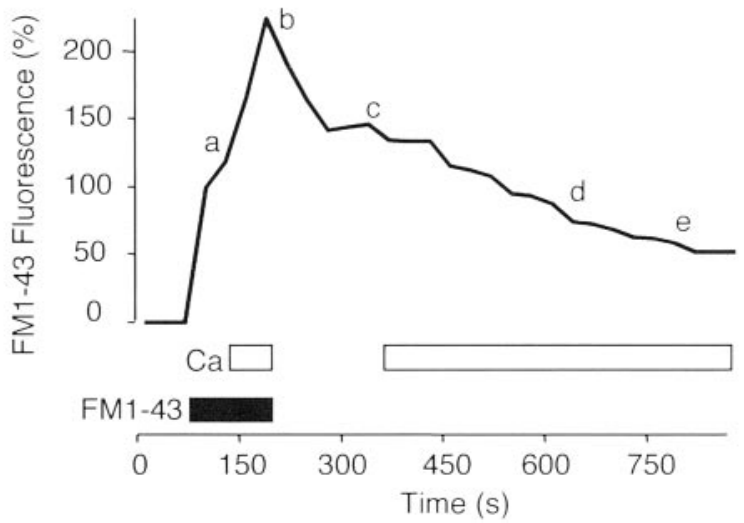

Figure 9. $\mathrm{Ca}^{2+}$ influx stimulated movement of vacuoles away from the plasma membrane. $A$, Movement of large membrane compartments loaded with FM1-43 is shown. Membrane turnover was stimulated for $1 \mathrm{~min}$ in the presence of FM1 $-43(b)$, and then $\mathrm{Ca}^{2+}$ and FM1-43 were removed $(c) . \mathrm{Ca}^{2+}$ was then reapplied in the presence of $50 \mathrm{~mm} \mathrm{~K}^{+}$to release FM1-43 from vesicles available for exocytosis $(d, e)$. The compartments containing nonreleasable FM1-43 were organized as a ring concentric with the plasma membrane $\sim 2 \mu \mathrm{m}$ from the surface. Scale bar, $10 \mu \mathrm{m}$. B, Timecourse of the fluorescence change in the synaptic terminal shown in $A$. Letters show the timing of corresponding images. Note that image e was obtained after complete loss of releasable FM1- 43 .

additional 5 min of stimulation (c). The arrows mark the position of the plasma membrane, obtained from simultaneously acquired DIC images. Labeled compartments stayed close to the surface in $0 \mathrm{Ca}^{2+}$ but moved away during stimulation. This is shown clearly by image subtraction. In Figure $10 \mathrm{Ba}$, the image obtained immediately after loading was subtracted from the image obtained at the end of the incubation in $0 \mathrm{Ca}^{2+}$. The result is almost uniformly black, indicating that there was very little movement of vacuoles during this time. Repeating the procedure by subtracting the image obtained in $0 \mathrm{Ca}^{2+}$ from the image obtained after depolarizing the cell in $2.5 \mathrm{mM} \mathrm{Ca}^{2+}$ for $5 \mathrm{~min}$ illustrates the directed movement of vacuoles toward the center of the terminal (Fig. $10 \mathrm{Bb}$ ).

The averaged timecourse of vacuole movements is plotted in Figure 10C. Immediately after loading with dextran, labeled compartments were located $\sim 0.5 \mu \mathrm{m}$ from the plasma membrane and did not move for periods of minutes in the absence of external $\mathrm{Ca}^{2+}$. Re-establishing $\mathrm{Ca}^{2+}$ influx by addition of $2.5 \mathrm{~mm}$ $\mathrm{Ca}^{2+}$ caused a radial movement of vacuoles to a mean distance of $\sim 1.6 \mu \mathrm{m}$ from the plasma membrane, and this movement had a time constant of $\sim 150 \mathrm{sec}$. The rate and extent of vacuole movement was very similar to the extension of the actin network measured by Job and Lagnado (1998). To test whether this was a causal relationship, we inhibited actin polymerization. When 20 $\mu \mathrm{M}$ latrunculin B was applied immediately after labeling com-
A
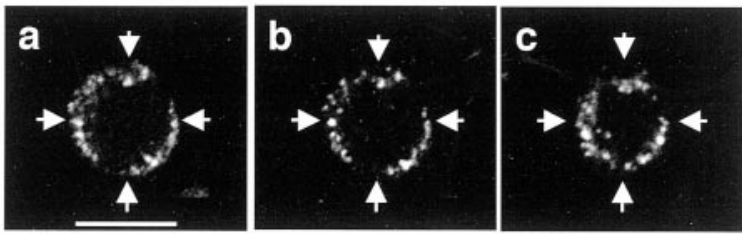

B
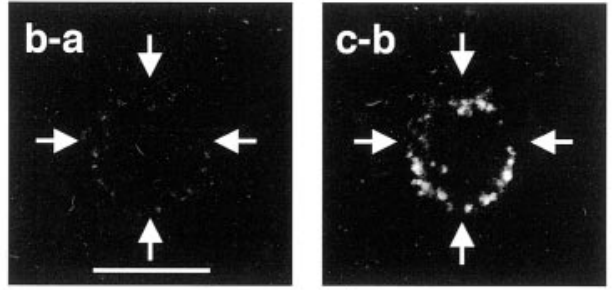

C

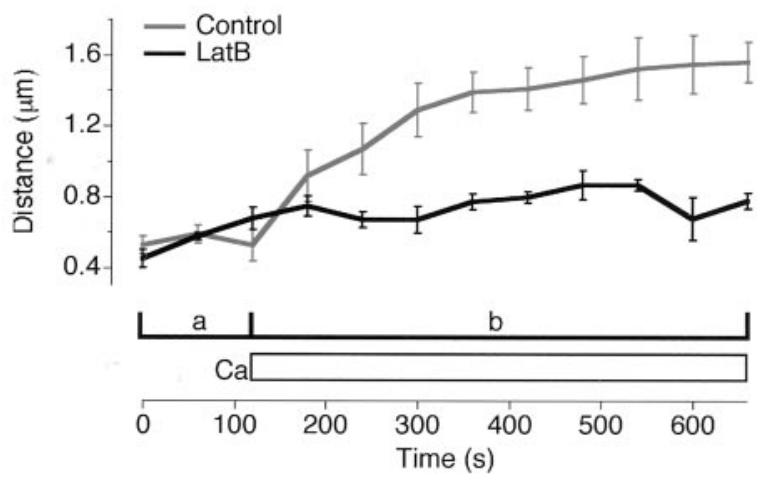

Figure 10. Movement of vacuoles was dependent on $\mathrm{Ca}^{2+}$ influx and actin polymerization. $A$, A series of confocal images from a terminal in which vacuoles were labeled with $40 \mathrm{kDa}$ dextran is shown. The position of labeled compartments is shown immediately after $1 \mathrm{~min}$ of stimulation ( $a$ ), after 2 min in $\mathrm{OCa}^{2+}(b)$, and after an additional 5 min of stimulation ( $(c)$. The arrows mark the position of the plasma membrane. Labeled compartments stayed close to the plasma membrane surface in $0 \mathrm{Ca}^{2+}$ but moved away during stimulation. Scale bar, $10 \mu \mathrm{m} . B$, Movement of labeled compartments highlighted by image subtraction is shown. When image $a$ is subtracted from image $b$, the result is almost uniformly black, indicating that there was very little movement of vacuoles during the period in $0 \mathrm{Ca}^{2+}$. When image $b$ is subtracted from image $c$, the inward movement of vacuoles in response to $\mathrm{Ca}^{2+}$ influx is apparent. Arrows mark the position of the plasma membrane, as in $A$. Scale bar, $10 \mu \mathrm{m}$. C, Collected results quantifying movement of vacuoles away from the plasma membrane under control conditions ( gray). This movement was blocked when $20 \mu \mathrm{m}$ latrunculin B was applied immediately after loading vacuoles with dextrans (black). Brackets a and $\mathrm{b}$ illustrate the periods of depolarization in $\mathrm{CCa}^{2+}$ and $2.5 \mathrm{~mm} \mathrm{Ca}{ }^{2+}$ referred to in A. Results are averaged from five cells.

partments with $40 \mathrm{kDa}$ dextran, subsequent $\mathrm{Ca}^{2+}$ influx failed to stimulate vacuole movement away from the plasma membrane (Fig. $10 \mathrm{~B}$ ). We conclude that the movement of vacuoles was dependent on actin polymerization stimulated by $\mathrm{Ca}^{2+}$ influx.

\section{Discussion}

These results demonstrate that $\mathrm{Ca}^{2+}$-triggered exocytosis in the synaptic terminal of retinal bipolar cells is followed by compensatory endocytosis into at least two compartments: small vesicles and large vacuoles. Retrieval and movement of these large endocytic compartments was dependent on actin dynamics and the activity of PI 3-kinase, but endocytosis of small vesicles was not. 


\section{Bulk membrane retrieval occurred by a process analogous to macropinocytosis}

Several properties of bulk membrane retrieval at this synapse were similar to macropinocytosis, an actin-dependent mechanism of endocytosis observed in a number of non-neural cells and especially well characterized in macrophages and dendritic cells (Swanson and Watts, 1995; Araki et al., 1996; West et al., 2000). The key points of similarity were: an association with membrane ruffling (Fig. 1), large compartments of heterogeneous size (Fig. 2), dependence on actin polymerization (Fig. 7), dependence on PI 3-kinase activity (Fig. 7), and movement of these compartments away from the surface membrane driven by actin polymerization (Figs. 9, 10). Macropinocytosis, like other mechanisms of bulk membrane retrieval, does not involve the formation of clathrin-coats on the membrane (Lamaze and Schmid, 1995; Nichols and Lippincott-Schwartz, 2001). The results we have presented do not rule out the possibility that other clathrin-independent mechanisms also operate at this synapse. In particular, it has not been established whether fast retrieval of small synaptic vesicles involves clathrin.

Retrieval into small vesicles could be distinguished from macropinocytosis in the synaptic terminal by several criteria, including exclusion of large dextrans (Fig. 2), wider distribution within the terminal and participation in the vesicle cycle (Figs. 1, 9), and lack of sensitivity to drugs that blocked actin polymerization (Fig. 7). The retinal bipolar cell is therefore qualitatively similar to a number of other synapses where small vesicles are retrieved in parallel with large invaginations and cisternas (Takei et al., 1996; Gad et al., 1998; Roos and Kelly, 1999; Richards et al., 2000; Teng and Wilkinson, 2000). It will be interesting to establish whether bulk membrane retrieval at these other synapses is also dependent on actin dynamics and PI 3-kinase activity.

\section{What is the role of macropinocytosis in the synaptic terminal of bipolar cells?}

Several observations indicated that macropinocytosis did not play an essential role in the continuous vesicle cycle that supports tonic release of neurotransmitter by this ribbon synapse. Macropinosomes did not release their contents (Fig. 3) and did not mix their contents with small vesicles (Fig. 4); continuous vesicle cycling continued at normal rates when macropinocytosis was completely inhibited by the PI 3-kinase inhibitor LY294002 (Fig. 7). In addition, macropinocytosis occurred predominantly during the initial minute of maintained stimulation, although vesicle cycling continued at a constant rate for many minutes (Fig. 5). These observations indicate that endocytosis into small vesicles alone was sufficient to balance and maintain exocytosis during continuous stimulation. In direct contrast to macropinocytosis, the endosomes formed at the neuromuscular junction occur only after a period of prolonged activity, and the number of endosomes increases for the duration of the stimulus (Heuser and Reese, 1973; Teng et al., 1999).

We suggest that macropinocytosis might be involved in the structural and functional plasticity of this synaptic terminal in the retina. The class of bipolar cell we used in this study normally receives inputs from rod photoreceptors, and the terminals extend spinules that invaginate the processes of amacrine cells. These spinules retract in response to depolarization by light (Yazulla and Studholme, 1992; Behrens and Wagner, 1996), and spinule retraction correlates with the growth of the cortical F-actin network (Job and Lagnado, 1998). Electron microscopy has demonstrated that spinules contain active zones (von Gersdorff et al., 1996), and their retraction causes the breaking of reciprocal synaptic connections between bipolar cells and amacrine cells (Yazulla and Studholme, 1992). It therefore seems that the structural plasticity of this synaptic terminal contributes to the phenomenon of "network adaptation" (i.e., the large fall in the gain of synaptic transmission through the retina that occurs in response to an increase in the mean level of illumination) (Dowling, 1987; Smirnakis et al., 1997; Brown and Masland, 2001). The bulk membrane retrieval that we have described seems a likely candidate for the process that causes spinule retraction when the bipolar cell depolarizes in response to light.

\section{The actin cytoskeleton and compensatory endocytosis at the synapse}

It has not been clear how actin might be involved in endocytosis at the synapse (Qualmann et al., 2000; Slepnev and De Camilli, 2000). Clathrin and actin colocalize at the endocytic regions that surround active zones of exocytosis (Roos and Kelly, 1999; Dunaevsky and Connor, 2000), and a number of proteins involved in the formation of coated pits and membrane fission interact with the actin cytoskeleton (Qualmann and Kelly, 2000). Recycling of vesicles at the reticulospinal synapse of lamprey is clathrin dependent and affected by perturbation of the actin cytoskeleton (Shupliakov et al., 2002). In contrast, we found that disruption of the actin cytoskeleton using cytochalasin D and latrunculin B did not affect retrieval of small vesicles in the synaptic terminal of bipolar cells, either during maintained stimulation (Fig. 7) or immediately after a brief stimulus (Fig. 8). These results cannot be interpreted as evidence against a role for actin in clathrin-mediated endocytosis, because it is not known how far retrieval of small vesicles in the synaptic terminal of bipolar cells might be mediated by formation of clathrin-coated pits. An additional complication is that drugs affecting the actin cytoskeleton have been found to have inconsistent effects on retrieval that is known to be clathrin dependent, such as receptor-mediated endocytosis (Fujimoto et al., 2000).

Agents that disrupted the actin cytoskeleton also had no discernible effect on continuous exocytosis during maintained stimulation (Fig. 7) or on fast exocytosis triggered by a $20 \mathrm{msec}$ depolarization (Fig. $8 \mathrm{~A}$ ) or by resupply of vesicles into the rapidly releasable pool (Fig. 8B,C). In comparison, Cole et al. (2000) found that actin facilitated the mobilization of vesicles at the snake neuromuscular junction during high levels of nerve terminal activity. This difference might be explained if the ribbon synapses of goldfish bipolar cells lack synapsin I, as do the ribbon synapses of many other species (Mandell et al., 1990; von Kriegstein et al., 1999). Synapsin I is associated with vesicles at most synapses and also binds actin filaments (Greengard et al., 1993).

Many proteins that regulate actin dynamics also bind to phosphoinositides in the plasma membrane (Cremona and De Camilli, 2001). Here we provide evidence that the generation of D3 phosphoinositides, via PI 3-kinase activity, is an important regulator of actin dynamics in the synaptic terminal of the bipolar cell. There are a number of possible mechanisms by which PI 3-kinase activity may regulate the actin cytoskeleton. D3 phosphoinositides bind to a number of proteins that regulate actin assembly, such as the actin-capping protein profilin and the actin-severing protein gelsolin. PI 3-kinase also regulates the activity of Rac, a small GTPase with an important role in actin remodeling (for review, see Toker and Cantley, 1997). Phosphoinositides are not uniformly distributed in cellular membranes (Pike, 1992), allowing them to act as site-specific signals for the recruitment and/or activation of multimolecular complexes on the membrane (Martin, 1998). It has been suggested that the generation of D3 
phosphoinositides by PI 3-kinase might recruit a large signaling complex at the plasma membrane that is essential for macropinocytosis (Clague et al., 1995; Toker and Cantley, 1997). The role of phosphoinositides in recruiting signaling molecules might also explain why LY294002 has the most profound effect on dextran uptake when compared with drug treatments that only target the actin cytoskeleton (latrunculin B and cytochalasin D). Actin polymerization may only be necessary to provide the mechanical force needed for the generation of the largest macropinosomes (Swanson and Watts, 1995).

Once formed, macropinosomes moved away from the plasma membrane surface in response to $\mathrm{Ca}^{2+}$ influx, and this process was dependent on actin polymerization (Figs. 9, 10). The rate and extent of this movement paralleled the extension of the actin network described by Job and Lagnado (1998). It may be that growing actin filaments push macropinosomes away from the surface membrane, perhaps by formation of actin comets (Merrifield et al., 1999; Orth et al., 2002). Alternatively, macropinosomes might travel on tracks provided by the actin cytoskeleton. Actin filaments are oriented with their pointed ends projecting away from the plasma membrane, whereas most myosins move toward the barbed end pointing into the membrane. However, myosin VI moves toward the pointed end (Wells et al., 1999) and might therefore "walk" macropinosomes into the cell.

In summary, this work provides a molecular characterization of macropinocytosis as a mechanism of compensatory endocytosis at the synapse. In the future, it will be important to determine which components of the surface and/or vesicle membrane are retrieved by this process.

Note added in proof. Sankaranarayanau et al. (2003) have used latrunculin A to disperse F-actin in synaptic boutons of hippocampal neurons in culture and also found no effect on the exocytosis or endocytosis of synaptic vesicles.

\section{References}

Araki N, Johnson MT, Swanson JA (1996) A role for phosphoinositide 3-kinase in the completion of macropinocytosis and phagocytosis by macrophages. J Cell Biol 135:1249-1260.

Behrens UD, Wagner HJ (1996) Adaptation-dependent changes of bipolar cell terminals in fish retina: effects on overall morphology and spinule formation in Ma and Mb cells. Vision Res 36:3901-3911.

Berthiaume EP, Medina C, Swanson JA (1995) Molecular size-fractionation during endocytosis in macrophages. J Cell Biol 129:989-998.

Brown SP, Masland RH (2001) Spatial scale and cellular substrate of contrast adaptation by retinal ganglion cells. Nat Neurosci 4:44-51.

Burrone J, Lagnado L (2000) Synaptic depression and the kinetics of exocytosis in retinal bipolar cells. J Neurosci 20:568-578.

Clague MJ, Thorpe C, Jones AT (1995) Phosphatidylinositol 3-kinase regulation of fluid phase endocytosis. FEBS Lett 367:272-274.

Cole JC, Villa BR, Wilkinson RS (2000) Disruption of actin impedes transmitter release in snake motor terminals. J Physiol (Lond) 525:579-586.

Cremona O, De Camilli P (2001) Phosphoinositides in membrane traffic at the synapse. J Cell Sci 114:1041-1052.

Crowder RJ, Freeman RS (1999) The survival of sympathetic neurons promoted by potassium depolarization, but not by cyclic AMP, requires phosphatidylinositol 3-kinase and Akt. J Neurochem 73:466-475.

Dowling JE (1987) The retina: an approachable part of the brain. Cambridge, MA: Harvard UP.

Dunaevsky A, Connor EA (2000) F-actin is concentrated in nonrelease domains at frog neuromuscular junctions. J Neurosci 20:6007-6012.

Ford MG, Pearse BM, Higgins MK, Vallis Y, Owen DJ, Gibson A, Hopkins CR, Evans PR, McMahon HT (2001) Simultaneous binding of PtdIns $(4,5) \mathrm{P}_{2}$ and clathrin by AP180 in the nucleation of clathrin lattices on membranes. Science 291:1051-1055.

Fried RC, Blaustein MP (1978) Retrieval and recycling of synaptic vesicle membrane in pinched-off nerve terminals (synaptosomes). J Cell Biol 78:685-700.
Fujimoto LM, Roth R, Heuser JE, Schmid SL (2000) Actin assembly plays a variable, but not obligatory role in receptor- mediated endocytosis in mammalian cells. Traffic 1:161-171.

Gad H, Low P, Zotova E, Brodin L, Shupliakov O (1998) Dissociation between $\mathrm{Ca}^{2+}$-triggered synaptic vesicle exocytosis and clathrin-mediated endocytosis at a central synapse. Neuron 21:607-616.

Gad H, Ringstad N, Low P, Kjaerulff O, Gustafsson J, Wenk M, Di Paolo G, Nemoto Y, Crun J, Ellisman MH, De Camilli P, Shupliakov O, Brodin L (2000) Fission and uncoating of synaptic clathrin-coated vesicles are perturbed by disruption of interactions with the $\mathrm{SH} 3$ domain of endophilin. Neuron 27:301-312.

Gomis A, Burrone J, Lagnado L (1999) Two actions of calcium regulate the supply of releasable vesicles at the ribbon synapse of retinal bipolar cells. J Neurosci 19:6309-6317.

Greengard P, Valtorta F, Czernik AJ, Benfenati F (1993) Synaptic vesicle phosphoproteins and regulation of synaptic function. Science 259:780-785.

Heuser JE, Reese TS (1973) Evidence for recycling of synaptic vesicle membrane during transmitter release at the frog neuromuscular junction. J Cell Biol 57:315-344.

Hirokawa N, Sobue K, Kanda K, Harada A, Yorifuji H (1989) The cytoskeletal architecture of the presynaptic terminal and molecular structure of synapsin 1. J Cell Biol 108:111-126.

Ikegami K, Koike T (2000) Membrane depolarization-mediated survival of sympathetic neurons occurs through both phosphatidylinositol 3-kinase and CaM kinase II-dependent pathways. Brain Res 866:218-226.

Job C, Lagnado L (1998) Calcium and protein kinase C regulate the actin cytoskeleton in the synaptic terminal of retinal bipolar cells. J Cell Biol 143:1661-1672.

Koenig J, Ikeda K (1989) Disappearance and reformation of synaptic vesicle membrane upon transmitter release observed under reversible blockage of membrane retrieval. J Neurosci 9:3844-3860.

Koenig J, Ikeda K (1996) Synaptic vesicles have two distinct recycling pathways. J Cell Biol 135:797-808.

Lagnado L, Gomis A, Job C (1996) Continuous vesicle cycling in the synaptic terminal of retinal bipolar cells. Neuron 17:957-967.

Lamaze C, Schmid SL (1995) The emergence of clathrin-independent pinocytic pathways. Curr Opin Cell Biol 7:573-580.

Mandell JW, Townes-Anderson E, Czernik AJ, Cameron R, Greengard P, De Camilli P (1990) Synapsins in the vertebrate retina: absence from ribbon synapses and heterogeneous distribution among conventional synapses. Neuron 5:19-33.

Martin TF (1998) Phosphoinositide lipids as signaling molecules: common themes for signal transduction, cytoskeletal regulation, and membrane trafficking. Annu Rev Cell Dev Biol 14:231-264.

Mennerick S, Matthews G (1996) Ultrafast exocytosis elicited by calcium current in synaptic terminals of retinal bipolar neurons. Neuron 17:1241-1249.

Merrifield CJ, Moss SE, Ballestrem C, Imhof BA, Giese G, Wunderlich I, Almers W (1999) Endocytic vesicles move at the tips of actin tails in cultured mast cells. Nat Cell Biol 1:72-74.

Merrifield CJ, Feldman ME, Wan L, Almers W (2002) Imaging actin and dynamin recruitment during invagination of single clathrin-coated pits. Nat Cell Biol 4:691-698.

Miller TM, Tansey MG, Johnson Jr EM, Creedon DJ (1997) Inhibition of phosphatidylinositol 3-kinase activity blocks depolarization- and insulinlike growth factor I-mediated survival of cerebellar granule cells. J Biol Chem 272:9847-9853.

Morales M, Colicos MA, Goda Y (2000) Actin-dependent regulation of neurotransmitter release at central synapses. Neuron 27:539-550.

Morton WM, Ayscough KR, McLaughlin PJ (2000) Latrunculin alters the actin-monomer subunit interface to prevent polymerization. Nat Cell Biol 2:376-378.

Neves G, Lagnado L (1999) The kinetics of exocytosis and endocytosis in the synaptic terminal of goldfish retinal bipolar cells. J Physiol (Lond) 515:181-202.

Neves G, Gomis A, Lagnado L (2001) Calcium influx selects the fast mode of endocytosis in the synaptic terminal of retinal bipolar cells. Proc Natl Acad Sci USA 98:15282-15287.

Nichols BJ, Lippincott-Schwartz J (2001) Endocytosis without clathrin coats. Trends Cell Biol 11:406-412.

Orth JD, Krueger EW, Cao H, McNiven MA (2002) From the cover: the large GTPase dynamin regulates actin comet formation and movement in living cells. Proc Natl Acad Sci USA 99:167-172. 
Osborne SL, Meunier FA, Schiavo G (2001) Phosphoinositides as key regulators of synaptic function. Neuron 32:9-12.

Pike LJ (1992) Phosphatidylinositol 4-kinases and the role of polyphosphoinositides in cellular regulation. Endocr Rev 13:692-706.

Qualmann B, Kelly RB (2000) Syndapin isoforms participate in receptormediated endocytosis and actin organization. J Cell Biol 148:1047-1062.

Qualmann B, Kessels MM, Kelly RB (2000) Molecular links between endocytosis and the actin cytoskeleton. J Cell Biol 150:F111-F116.

Richards DA, Guatimosim C, BetzWJ (2000) Two endocytic recycling routes selectively fill two vesicle pools in frog motor nerve terminals. Neuron 27:551-559.

Roos J, Kelly RB (1999) The endocytic machinery in nerve terminals surrounds sites of exocytosis. Curr Biol 9:1411-1414.

Rouze NC, Schwartz EA (1998) Continuous and transient vesicle cycling at a ribbon synapse. J Neurosci 18:8614-8624.

Sankaranarayanau S, Atluri PP, Ryan TA (2003) Actin has a molecular scaffolding, not propulsive, role in presynaptic function. Nat Neurosci, in press.

Shupliakov O, Bloom O, Gustafsson JS, Kjaerulff O, Low P, Tomilin N, Pieribone VA, Greengard P, Brodin L (2002) Impaired recycling of synaptic vesicles after acute perturbation of the presynaptic actin cytoskeleton. Proc Natl Acad Sci USA 99:14476-14481.

Slepnev VI, De Camilli P (2000) Accessory factors in clathrin-dependent synaptic vesicle endocytosis. Nat Rev Neurosci 1:161-172.

Smirnakis SM, Berry MJ, Warland DK, Bialek W, Meister M (1997) Adaptation of retinal processing to image contrast and spatial scale. Nature 386:69-73.

Swanson JA, Watts C (1995) Macropinocytosis. Trends Cell Biol 5:424-428.

Takei K, Mundigl O, Daniell L, De Camilli P (1996) The synaptic vesicle cycle: a single vesicle budding step involving clathrin and dynamin. J Cell Biol 133:1237-1250.

Teng H, Wilkinson RS (2000) Clathrin-mediated endocytosis near active zones in snake motor boutons. J Neurosci 20:7986-7993.
Teng H, Cole JC, Roberts RL, Wilkinson RS (1999) Endocytic active zones: hot spots for endocytosis in vertebrate neuromuscular terminals. J Neurosci 19:4855-4866.

Toker A, Cantley LC (1997) Signalling through the lipid products of phosphoinositide-3-OH kinase. Nature 387:673-676.

Vlahos CJ, Matter WF, Hui KY, Brown RF (1994) A specific inhibitor of phosphatidylinositol 3-kinase, 2-(4-morpholinyl)-8-phenyl-4H-1-benzopyran4-one (LY294002). J Biol Chem 269:5241-5248.

von Gersdorff H, Matthews G (1994) Dynamics of synaptic vesicle fusion and membrane retrieval in synaptic terminals. Nature 367:735-739.

von Gersdorff H, Vardi E, Matthews G, Sterling P (1996) Evidence that vesicles on the synaptic ribbon of retinal bipolar neurons can be rapidly released. Neuron 16:1221-1227.

von Kriegstein K, Schmitz F, Link E, Sudhof TC (1999) Distribution of synaptic vesicle proteins in the mammalian retina identifies obligatory and facultative components of ribbon synapses. Eur J Neurosci 11:1335-1348.

Wells AL, Lin AW, Chen LQ, Safer D, Cain SM, Hasson T, Carragher BO Milligan RA, Sweeney HL (1999) Myosin VI is an actin-based motor that moves backwards. Nature 401:505-508.

West MA, Prescott AR, Eskelinen EL, Ridley AJ, Watts C (2000) Rac is required for constitutive macropinocytosis by dendritic cells but does not control its downregulation. Curr Biol 10:839-848.

Yazulla S, Studholme KM (1992) Light-dependent plasticity of the synaptic terminals of $\mathrm{Mb}$ bipolar cells in goldfish retina. J Comp Neurol 320:521-530.

Zenisek D, Steyer JA, Almers W (2000) Transport, capture and exocytosis of single synaptic vesicles at active zones. Nature 406:849-854.

Zenisek D, Steyer JA, Feldman ME, Almers W (2002) A membrane marker leaves synaptic vesicles in milliseconds after exocytosis in retinal bipolar cells. Neuron 35:1085-1097. 